Trauma Berufskrankh 2008 · 10[Suppl 1]:25-31 DOI 10.1007/s10039-007-1279-3

Online publiziert: 29. November 2007

c) Springer Medizin Verlag 2007

M.H. Amlang ${ }^{1} \cdot$ M. Schmidt ${ }^{2} \cdot$ W. Röhnert ${ }^{3} \cdot$ F. Czornack ${ }^{1}$ H. Zwipp ${ }^{1}$ C. Dahlen ${ }^{2}$

${ }^{1}$ Klinik für Unfall- und Wiederherstellungschirurgie, Universitätsklinikum „Carl Gustav Carus“, Technische Universität Dresden

${ }^{2}$ Klinik für Unfall- und Wiederherstellungschirurgie, Städtisches Krankenhaus Dresden-Neustadt, Dresden

${ }^{3}$ Radiologische Praxis, Döbeln

\title{
Proximale Humerusfraktur
}

\section{Konservativ-funktionelle Therapie nach Poelchen}

Die konservativ-funktionelle Therapie der proximalen Humerusfraktur nach Poelchen war in Sachsen bis Anfang der 1990er Jahre die am weitesten verbreitete Behandlungsform. Durch die demografische Entwicklung der deutschen Bevölkerung und die daraus folgende Zunahme der proximalen Humerusfrakturen im hohen Lebensalter, das hohe Operationsrisiko beim sehr alten Patienten und die ökonomischen Zwänge im Gesundheitswesen wird die Frage nach der Indikation zur konservativen Therapie der proximalen Humerusfraktur wieder aktuell.

\section{Geschichte der so genannten "Poelchen-Behandlung"}

Der Chirurg Richard Poelchen arbeitete 1878 in der chirurgischen Nebenabteilung der Berliner Charitè, die von einem Oberstabsarzt Starcke geleitet wurde. Dort sah er die ausschließlich funktionelle Frakturbehandlung ohne ,jeden feststellenden" Verband, die Starcke im deutschfranzösischen Krieg 1871/1872 in den Lazaretten von Metz, die der Kieler Chirurg von Esmarch leitete, gelernt hatte. Durch den Massenanfall von Verletzten konnte bei Frakturen häufig kein Gips angelegt werden, sondern die verletzte Extremität wurde nur durch Lagerung oder überhaupt nicht behandelt. Von Esmarch stellte zu seiner Überraschung fest, dass die nach damaligen Maßstäben nur ungenügend retinierten Frakturen häufig bessere funktionelle Ergebnisse aufwiesen als die mit einer korrekten Gipsbehandlung. Von Esmarch hatte aber selbst nie etwas über diese Beobachtungen veröffentlicht.

Richard Poelchen wurde nach seiner Ausbildung Oberarzt und später Chefarzt des Krankenhauses in Zeitz, einer kleinen Stadt in Sachsen-Anhalt, die damals zu Preußen gehörte und bis 1815 sächsisch war. Er widmete sich bei seiner chirurgischen Arbeit v. a. der Versorgung Unfallverletzter und musste feststellen, dass die damals vorherrschende Unfallheilkunde nach den Richtlinien von Lorenz Böhler mit der Perfektionierung der Gipsbehandlung und der damit verbundenen Immobilisation erhebliche Nachteile besonders bei der funktionellen Wiederherstellung aufwies. Er erkannte, dass eine frühfunktionelle Behandlung in vielen Fällen Vorteile gegenüber der immobilisierenden Therapie aufwies. Besonders die Reintegration in den Beruf war damals bei der kaum vorhandenen sozialen Absicherung entscheidend für das weitere Schicksal des Verletzten und nicht zuletzt auch seiner Familie. So richtete Poelchen in seiner Praxis einen Raum ein, in dem die Arbeitsfähigkeit von Patienten mit Armverletzungen durch frühestmöglichen, therapeutischen Gebrauch von Werkzeugen wiederhergestellt wurde. Er ist damit einer der Väter der Ergotherapie. Mit der Behandlung von Knochenbrüchen durch frühfunktionelle Behandlung unter Verzicht auf eine Immobilisa- tion und möglichst anatomische Reposition stand er im Widerspruch zur Böhler-Schule, bei der die korrekte Reposition und Retention der Fraktur bis zur knöchernen Konsolidierung im Mittelpunkt standen. Erst durch die moderne Unfallchirurgie der 1970er Jahre mit der übungsstabilen Osteosynthese konnte dieser Widerspruch aufgelöst werden.

Poelchen war zu seiner Zeit ein Außenseiter, der sich erst am Ende seines Berufslebens entschlossen hatte, seine Er-

\section{Selbstinnervationsbehandlung geschlossener Knochenbrüche und Verrenkungen, eine biologische Behandlungsart}

Geh. San.-Rat Dr. med. R:chard Poelchen

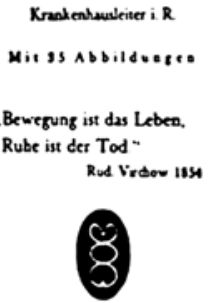

HIPPOKRATES.VERLAG MARQUARDT \& CIE. STUTTGART

Abb. 1 A Im Jahr 1940 von Richard Poelchen unter dem Motto Virchows „Bewegung ist Leben, Ruhe ist Tod." im Hippokrates-Verlag veröffentlichtes Buch 


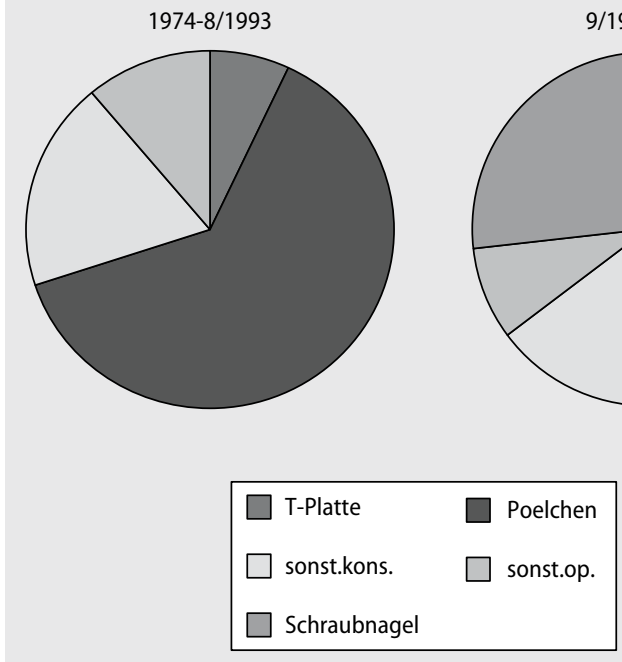

9/1993-1995

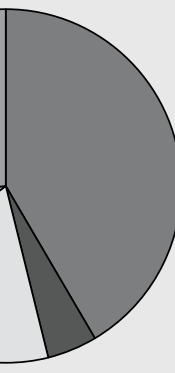

fahrungen niederzuschreiben. Im Jahr 1940 veröffentlichte er sein Buch mit dem Virchow-Zitat als Motto „Bewegung ist Leben, Ruhe ist der Tod“ (• Abb. 1) [22]. Darin grenzte er sich auch zur Frakturbehandlung mit passiver Bewegungstherapie und Massage von Lukas-Championnière $\mathrm{ab}$ [22]. Er wurde von den zu dieser Zeit „meinungsbildenden“ Chirurgen ignoriert oder belächelt. Die Ironie der Geschichte ist, dass im gleichen Jahr noch ein anderer Wegbereiter der modernen Unfallchirurgie mit seiner bahnbrechenden Idee der intramedullären Frakturschienung von der Elite der deutschen Chirurgie abgelehnt wurde, der Sachse Gerhard Küntscher. Er stand allerdings am Anfang seiner chirurgischen Laufbahn und hatte noch das Glück, den Durchbruch seiner genialen Methode zu erleben.

Eine weitere Brücke kann man in die heutige Zeit und zu einer aktuellen Aufgabe der Unfallchirurgie schlagen, dem $\mathrm{Zu}$ sammenwachsen der Unfallchirurgie mit der Orthopädie. Das Buch von Poelchen wäre dem Vergessen anheim gefallen, wenn nicht der Berliner Ordinarius für Orthopädie, Hermann Gocht, die Bedeutung der frühfunktionellen Behandlung nach Poelchen bei Verletzungen der oberen Extremität erkannt, unterstützt und in der eigenen Klinik praktiziert hätte. Gocht schrieb das Geleitwort für Poelchens Buch und verlieh diesem damit die notwendige Legitimation, sodass sich die Methode im klinischen Alltag etablieren konnte. Nach seiner Meinung zeichnete sich die frühfunktionelle Behandlung nach Poelchen durch 5 Merkmale aus:
“1. in der Stärkung des Willens und der Zuversicht des Verletzten von vornherein,

2. in ihrer möglichst schmerzlosen und seelisch nicht belastenden Einleitung und Durchführung,

3. in ihrer Einfachheit,

4. in der Kürze der Behandlungszeit,

5. in ihren guten, endgültigen Ergebnissen bezüglich der wiedererreichten Funktion und damit der Leistungsfähigkeit. „ [22]

Dass Richard Poelchen nicht dogmatisch bei der Umsetzung seiner frühfunktionellen Behandlung unter weitgehendem Verzicht auf die Reposition war, kommt in folgender Passage der Einleitung Gochts zum Ausdruck:

“... Poelchen [ist] dieser Einseitigkeit nie verfallen, er nimmt bei besonders ungünstigen Knochenverschiebungen die einmalige, genaue Brucheinstellung vor, er wird in Notfällen dem operativen Vorgehen gerecht, verwirft auch die Gipsschiene nicht ein für allemal.“ [22].

\section{„Poelchen-Behandlung" der proximalen Humerusfraktur}

Die klassische Poelchen-Behandlung der proximalen Humerusfraktur ist die möglichst frühe, aktive Bewegung des Arms („Selbstinnervation“ nach Poelchen) in Form von Pendelübungen, wobei ein Gewicht von etwa $15 \mathrm{~N}(1,5 \mathrm{kp})$ mit einer Schlinge am Handgelenk befestigt wird. Durch den Zug des Gewichts kommt es zu einer Schmerzreduktion und zur Straffung der Weichteile der Schulter, wodurch eine Schienung der Fraktur erreicht wird, wie es auch Sarmiento für die Oberarmund Unterschenkelschaftfraktur mit Hilfe eines Brace beschrieben hat. Die früher regelmäßig angelegte Gipslonguette mit Schultereinschluss, die auch zur Extension eingesetzt wurde und eine vorzeitige Abduktion des Arms verhindern sollte, bezeichnete man fälschlicherweise als Poelchen-Gips. Poelchen hatte nie eine derartige Gipsschiene empfohlen, und sie wurde wahrscheinlich als Zugeständnis an die weit verbreitete Extensionsbehandlung Teil der Therapie.

\section{Material und Methode}

Im Rahmen einer Promotion [28] wurden 313 Patienten mit einer proximalen Humerusfraktur erfasst, die von 1974-1995 am Universitätsklinikum „Carl Gustav Carus" in Dresden (bis September 1993 Medizinische Akademie Dresden) behandelt wurden (- Abb. 2). Ausschlusskriterien waren:

- ein Alter zum Unfallzeitpunkt von weniger als 16 Jahren,

- Patienten mit pathologischen Frakturen,

- ein Alter zum Zeitpunkt der Einbestellung von mehr als 90 Jahren sowie

- ein weiter Anreiseweg zur Nachuntersuchung.

Es konnten 197 Patienten in die Studie eingeschlossen werden. Diese wurden angeschrieben, um die Beantwortung eines Fragebogens gebeten und zur Nachuntersuchung einbestellt. Die Fragen wurden von 123 Patienten $(62,4 \%$ ) (66 nach konservativer und 57 nach operativer Therapie) beantwortet. Von den 66 Patienten mit konservativer Therapie waren 46 (70\%) weiblich und 22 (30\%) männlich. Das Durchschnittsalter zum Unfallzeitpunkt betrug 57,9 Jahre (16-78 Jahre).

Von den 74 Patienten, die nicht in diese Studie einbezogen werden konnten, waren 31 unbekannt verzogen, 3 Pflegefälle, 16 bereits verstorben und 24 nicht zur Teilnahme an der Studie bereit.

Zur Nachuntersuchung erschienen 90 Patienten (45,7\%), davon 51 nach konservativer Therapie. Der mittlere Nachuntersuchungszeitraum betrug 52,8 Monate (6261 Monate). 


\section{Fragebogen}

In ihm wurden erfasst:

- Patientenzufriedenheit,

- Aktivitätseinschränkungen durch die Fraktur und

- die subjektiven Parameter des Constant- sowie des Neer-Scores.

\section{Klinisch-radiologische Nachuntersuchung}

Der Constant- und der Neer-Score wurden bestimmt. Außerdem wurden der Nackengriff, der Schürzengriff und der Kratztest nach Apley geprüft.

Die Unfallaufnahmen wurden hinsichtlich Qualität und Vollständigkeit beurteilt und der Frakturtyp nach Neer [20] bestimmt.

Bei der Nachuntersuchung wurden die betreffende Schulter in der a.-p. und axialen Ebene geröntgt und das Behandlungsergebnis nach den Kriterien des Neer-Scores beurteilt [20].

\section{Statistik}

Der Einfluss von Variablen auf das Ergebnis wurde mit dem $\chi^{2}$-Test geprüft. Es wurden das Konfidenzintervall mit $\alpha=0,05$ festgelegt, die Standardabweichung SD sowie die Irrtumswahrscheinlichkeit $\mathrm{p}$ bestimmt und das Signifikanzniveau mit 0,05 determiniert.

\section{Ergebnisse}

\section{Behandlungsmethoden}

Im Zeitraum 1974-8/1993 wurden 93\% der stationären Patienten konservativ behandelt, wobei mit $62 \%$ die so genannte „Poelchen-Behandlung" die bevorzugt verwendete Methode war ( Abb. 2). Im Zeitraum 9/1993-1995 ging der Anteil der konservativ behandelten Patienten auf $23 \%$ zurück, wobei nur noch bei $4 \%$ der Patienten explizit der Begriff der „Poelchen-Behandlung" gewählt wurde. Der überwiegende Teil der Patienten mit konservativer Behandlung wurde durch eine Ruhigstellung im Gilchrist-Verband behandelt.

Trauma Berufskrankh 2008 · 10[Suppl 1]:25-31 DOI 10.1007/s10039-007-1279-3

(c) Springer Medizin Verlag 2007

\section{M.H. Amlang · M. Schmidt · W. Röhnert · F. Czornack · H. Zwipp · C. Dahlen Proximale Humerusfraktur. Konservativ-funktionelle Therapie nach Poelchen}

\section{Zusammenfassung}

Die Ergebnisse der konservativ-funktionellen Therapie der proximalen Humerusfraktur nach Poelchen wurden bei 66 Patienten analysiert. Klinisch-radiologisch mit Bestimmung des Constant- und des Neer-Scores wurden 51 Patienten untersucht, von denen $74 \%(n=49)$ mit dem Behandlungsergebnis sehr zufrieden oder zufrieden, $20 \%(n=13)$ bedingt und $6 \%(n=4)$ nicht zufrieden waren. $64 \%(n=42)$ hatten keine oder nur leichte, $27 \%(n=18)$ mäßige und $9 \%(n=6)$ stärkere Schmerzen. $74 \%(n=49)$ gaben hinsichtlich der Auswirkungen der Beschwerden auf die Lebensgewohnheiten keine Beeinträchtigung an. Nach dem Constant-Score betrug der Punkteverlust gegenüber der gesunden Schulter 23 Punkte (0-82 Punkte, SD=22). Im Neer-Score erreichten $29 \%(n=15)$ ein ausgezeichnetes, $25 \%$ ( $n=13$ ) ein befriedigendes und $12 \%(n=6)$ ein unbefriedigendes $(12 \%)$ Ergebnis, bei $34 \%$ ( $n=17)$ war ein Misserfolg zu verzeichnen. Sowohl der Neer- als auch der Constant-Score korrelierten signifikant mit dem Ausmaß der radiologischen Veränderungen. Gerade beim sehr alten und multimorbiden Menschen ist die konservativ-funktionelle Therapie der proximalen Humerusfraktur nach Poelchen eine risikoarme Option mit überwiegend guten und befriedigenden Ergebnissen. Bei 4-Teile-Frakturen, Luxationsfrakturen und Kopfspaltbrüchen ist sie nur in Ausnahmefällen indiziert.

\section{Schlüsselwörter}

Proximale Humerusfraktur · Konservative Therapie · Poelchen · Humeruskopffraktur Geriatrie

\section{Proximal humerus fracture. Nonoperative functional therapy according to Richard Poelchen}

\section{Abstract}

Results of conservative functional therapy of fractures of the proximal humerus according to Poelchen were analysed in 66 patients. Clinical and X-ray examinations were conducted and the Constant and Neer scores determined in 51 of these patients; $74 \%(n=49)$ were satisfied or very satisfied with the outcome of treatment, while $20 \%(n=13)$ were partially satisfied and $6 \%(n=4)$ were not satisfied. Most (64\%; $n=42$ ) of the patients had only mild pain or none at all, while $27 \%$ $(n=18)$ reported moderate pain and $9 \%$ $(n=6)$ were suffering severe pain. When asked about any direct or indirect effects of their symptoms on their daily life, 49 (74\%) declared that they did not experience any restrictions. According to the Constant score, there was an average loss of 23 points $(0-82$ points, $S D=22$ ) compared with the healthy shoulder. According to the Neer score, 15 pa- tients (29\%) achieved an excellent outcome, $13(25 \%)$ a satisfactory result, $6(12 \%)$ an unsatisfactory result and 17 (34\%) a treatment failure. Both the Neer score and the Constant score correlated significantly with the extent of radiological alterations. Especially for very old and chronically ill people Nonoperative functional treatment of proximal fractures of the humerus is a safe option with predominantly good and satisfactory results precisely in very old patients and in those with multiple morbidities. It is indicated only in exceptional cases for treatment of four-part fractures, dislocated fractures and hairline fractures of the humeral head..

\section{Keywords}

Proximal fracture of the humerus .

Nonoperative therapy · Poelchen · Fracture of the humeral head · Geriatrics 


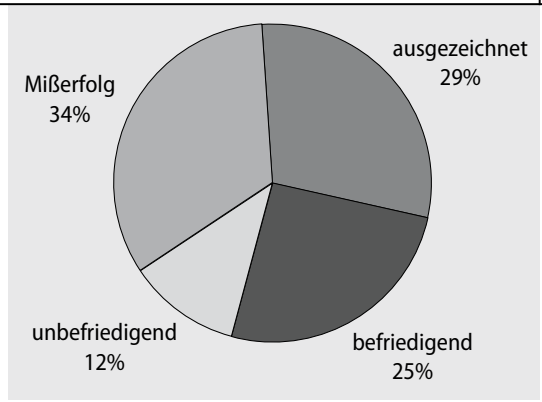

Abb. $3 \Delta$ Ergebnisse der konservativ behandelten Patienten nach dem Neer-Score $(n=51)$

\section{Auswertung des Fragebogens}

Schmerzen. Nach den Kriterien des Constant-Scores gaben 42 (64\%) der konservativ behandelten Patienten keine oder nur leichte Schmerzen an. 18 Patienten (27\%) klagten über mäßige und 6 ( $9 \%)$ über beträchtliche Schmerzen.

\section{Beeinträchtigung der Lebensgewohn-} heiten oder des Berufs. Bei der Frage nach den Auswirkungen der Beschwerden auf die Lebensgewohnheiten gaben 49 Patienten (74\%) keine Beeinträchtigung an. Nur 16 Patienten (24\%) berichteten über Beschwerden mit Beeinträchtigung der Lebensgewohnheiten oder des Berufs. In 1 Fall (2\%) führte der Unfall zur Aufgabe des Berufs.

Zufriedenheit. 49 Patienten (74\%) waren sehr zufrieden oder zufrieden mit dem Behandlungsergebnis. Nur bedingt zufrieden waren 13 (20\%) und nicht zufrieden 4 Patienten (6\%).

\section{Röntgenologische Klassifikation der Fraktur nach Neer}

In 36 Fällen (71\%) handelte es sich um die prognostisch günstigeren Typen I, III/2, IV/2, IV/3. Mit jeweils 1 Fall waren die Typen $\mathrm{II}, \mathrm{VI} / 3$ und $\mathrm{VI} / / 4$ vertreten.

Bei 12 (24\%) der konservativ behandelten Patienten war aufgrund schlechter Aufnahmequalität, Röntgenbild in nur einer Ebene oder fehlender Aufnahmen eine Klassifizierung nicht möglich.

\section{Klinisches Ergebnis}

Bewegungsumfang. Die Bewegungseinschränkung bei Elevation und Abduktion betrug im Mittel $33^{\circ}\left(\mathrm{o}-110^{\circ}, \mathrm{SD}=34,9\right)$. In
22 Fällen (43\%) zeigten sich Verminderungen der Elevation/Abduktion bis $10^{\circ}$, in 10 Fällen $(20 \%) 11-30^{\circ}$, bei $6(12 \%) 31-$ $60^{\circ}$ und bei $13(25 \%)$ mehr als $60^{\circ}$.

Funktionsfähigkeit. Im Neer-Score erreichten die konservativ behandelten $\mathrm{Pa}$ tienten bei der mit maximal 30 Punkten bewerteten Funktionsfähigkeit im Mittel 24,7 Punkte (8-30 Punkte, SD=5,9), wobei in diese Bewertung Kraft, Gebrauchspositionen des Arms und Stabilität eingingen $[20,30]$.

Subjektiver Teil des Constant-Scores. Es wurden im Mittel 26 (6-35 Punkte, SD=8) von maximal 35 Punkten erreicht, wobei Schmerzen und Alltagsaktivitäten berücksichtigt wurden [30].

Bewegungsausmaß und Kraft im Constant-Score. Es konnten im Mittel 42,7 (8-65 Punkte, $\mathrm{SD}=16,3)$ von maximal 65 Punkten erreicht werden, wobei das Bewegungsausmaß mit 40 Punkten und die Kraft mit 25 Punkten in die Beurteilung eingingen [30].

\section{Röntgenologisches Ergebnis}

Die Auswertung der Röntgenbilder zeigte entsprechend den Kriterien des NeerScores bei $12 \%$ keine, bei $39 \%$ geringe, bei $29 \%$ mäßige und bei $20 \%$ beträchtliche Veränderungen.

Bei der statistischen Überprüfung des Zusammenhangs zwischen der Schwere der Veränderungen im Röntgenbild und dem Ergebnis konnte sowohl für den $\mathrm{Ne}$ er- $(p<0,05)$ als auch für den ConstantScore $(p<0,05)$ ein statistisch signifikanter Zusammenhang gesichert werden.

\section{Gesamtergebnis}

Neer-Score. Bei der Beurteilung des Behandlungsergebnisses wurden im Mittel 73,7 Punkte (16-10o Punkte, SD=23) erreicht. Ein ausgezeichnetes Ergebnis erreichten 15 (29\%), ein befriedigendes 13 (25\%) und ein unbefriedigendes 6 Patienten (12\%). Bei 17 Patienten (34\%) musste ein Misserfolg konstatiert werden (- Abb. 3).
Constant-Score. Der Punkteverlust der verletzten gegenüber der gesunden Schulter betrug 23 Punkte (o-82 Punkte, $\mathrm{SD}=22$ ).

\section{Diskussion}

Richard Poelchen ist als Vater der frühfunktionellen Nachbehandlung von Frakturen und der Ergotherapie heute in Vergessenheit geraten [22]. Diese Arbeit soll zunächst diesen Chirurgen wieder in Erinnerung rufen und helfen, ihm den Platz in der chirurgischen Tradition zu geben, den er verdient. Gerade seine Tätigkeit als Durchgangsarzt für die gesetzliche Unfallversicherung in einer Zeit, in der eine soziale Absicherung bei Arbeitsunfähigkeit so gut wie nicht vorhanden war, führte ihn zu dem zukunftsweisenden Therapieansatz der Ergotherapie. Er machte die Erfahrung, dass die Gebrauchsfähigkeit der oberen Extremität am besten durch das spielerische Verrichten von gewohnten handwerklichen und berufsorientierten Arbeiten zu erreichen ist. Aus heutiger Sicht erscheint dieses Prinzip gerade als generelle Vorgehensweise auch bei instabilen Frakturen und unter Verzicht auf die Reposition undifferenziert und nicht mehr zeitgemäß. Es bildete aber den Gegenpol zu der damals herrschenden Böhler-Schule, die eine Frakturbehandlung durch Reposition und Retention durch Immobilisation in der Regel im Gipsverband propagierte. Die Intention von Poelchen, eine frühestmögliche, aktive Übungsbehandlung nach einer Fraktur zur Vermeidung von Immobilisationsschäden wie Muskelatrophie und Gelenkkontrakturen zu erreichen, ist erst durch die moderne, übungsstabile Osteosynthese möglich geworden.

Gerade für die Therapie der proximalen Humerusfraktur wurde nach dem 2. Weltkrieg in Deutschland die konservativ-funktionelle Therapie nach Poelchen ein etabliertes und breit eingesetztes Behandlungsverfahren. In den wegweisenden Arbeiten von Neer [20] wurde die konservative Therapie für die gering dislozierten Frakturen (1-Teil-Fraktur) als „Goldstandard“ definiert und durch mehrere Studien bestätigt $[1,12,15,33]$. Dabei sollte berücksichtigt werden, dass als $\mathrm{Ne}$ er-Kriterium für eine geringe Dislokation 
eine Angulation bis $45^{\circ}$ und eine Dislokation bis $1 \mathrm{~cm}$ definiert sind (- Abb. 4). Für die Dislokation des Tuberculum majus ist die tolerable Dislokation nach kranial später auf weniger als $5 \mathrm{~mm}$ korrigiert worden [10].

Obwohl sich das Konzept der Fragmentdefinition („part“) durch den Grad der Dislokation klinisch bewährt hat, ergeben sich hieraus auch die offensichtlichen Probleme bei der Reproduzierbarkeit der Neer-Klassifikation. Frakturlinien, die röntgenologisch auf eine Separation aller 4 Segmente nach Codman schließen lassen, begründen bei geringer Dislokation eben nicht die Einteilung als 4-Teile-Fraktur nach Neer. Die Klassifikation von Habermeyer $[5,6]$ hat sich trotz logischer Verbindung der Frakturmorphologie mit der zu erwartenden Prognose und besserer Reproduzierbarkeit leider nicht durchsetzen können. Nach Habermeyer $[5,6]$ sind es die nicht dislozierten Typ-o-Frakturen, die gut konservativ behandelt werden können.

Unsere Arbeit zeigt, dass bei einer undifferenzierten Indikationsstellung zur konservativen Therapie in etwa $1 / 3$ der Fälle mit einem schlechten Behandlungsergebnis zu rechnen ist. Schlechte Ergebnisse hatten v. a. Patienten mit:

- Spaltbruch des Humeruskopfs,

- Luxationsfraktur und

- 4-Teile-Fraktur.

Bereits in einer vor fast 30 Jahren veröffentlichten Arbeit von Weise et al. [32] wurde dies klar herausgearbeitet und hat nach wie vor Gültigkeit. Zu diesem Ergebnis kam auch Leyshon [17] in einer Studie an 42 Patienten mit konservativer Therapie, bei der alle 8 Fälle einer 4-Teile-Fraktur ein schlechtes Behandlungsergebnis aufwiesen. Keene et al. [12] konnten Analoges bestätigen. Inwieweit aber die Osteosynthese der 4-Teile-Fraktur signifikant bessere Ergebnisse bringt, ist bis heute noch nicht sicher geklärt. Die klassische Plattenosteosynthese mit der T-Platte erbringt nach eigenen Ergebnissen bei der 3- und 4-Teile-Fraktur keine besseren $\mathrm{Re}$ sultate als die konservative Therapie [28]. Dagegen wurde über gute Ergebnisse der minimalinvasiven Osteosynthese nach Resch von verschiedenen Arbeitsgruppen berichtet $[9,24,29]$. In einer rando-
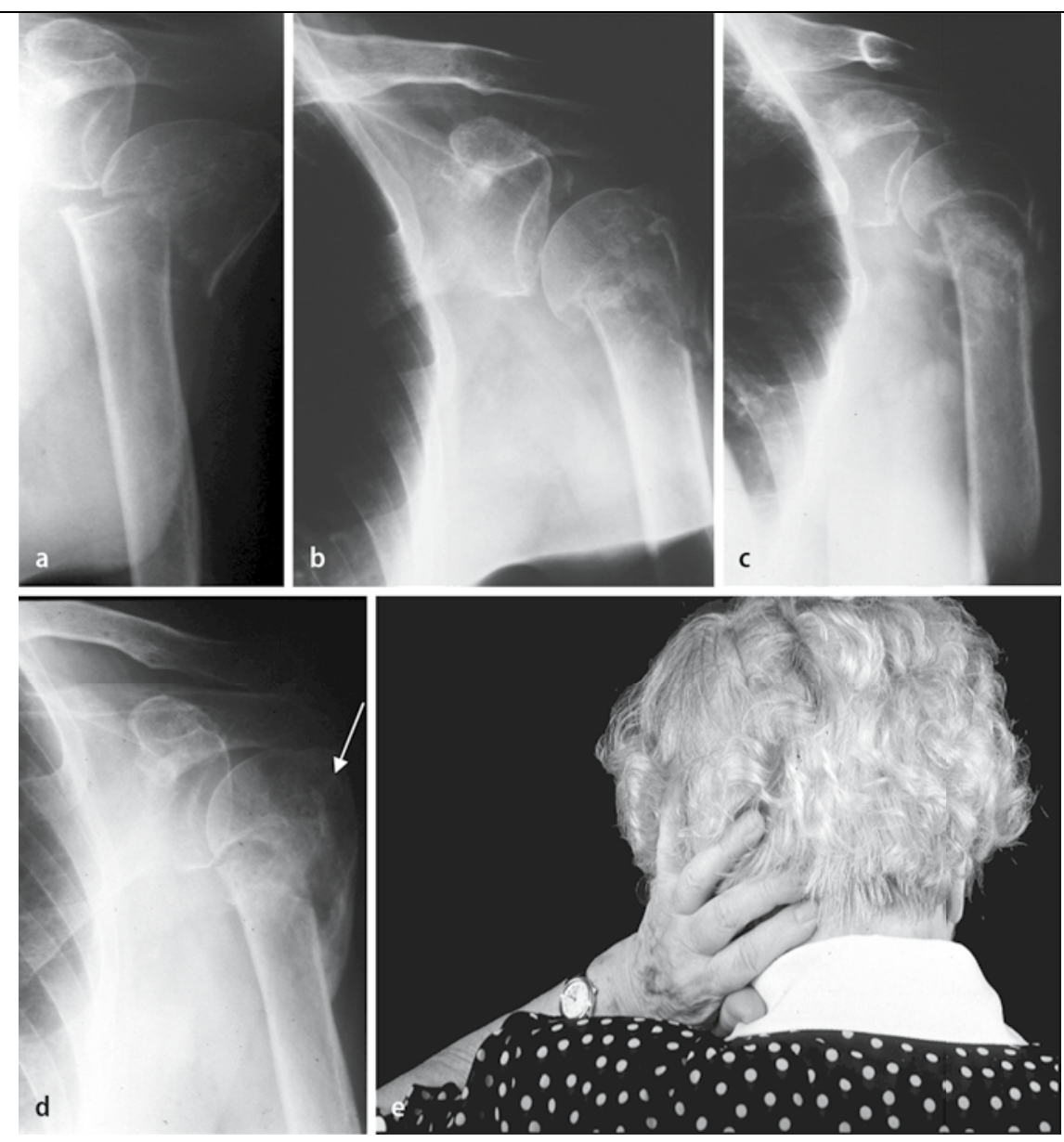

Abb. $4 \Delta$ a III/2-Fraktur nach Neer (instabil), b Reposition unter Bildwandlerkontrolle, c Röntgenkontrolle nach 3 Wochen frühfunktioneller Behandlung mit Zeichen der Instabilität und ausbleibender knöcherner Konsolidierung, deswegen Ruhigstellung im Gilchrist-Verband für 3 Wochen, danach geführte Bewegungen, $\mathbf{d}$ Röntgenkontrolle nach 8 Wochen mit Ausbildung einer lateralen Kallusbrücke (Pfeil), e funktionelles Ergebnis

misierten Studie von Zyto et al. [35] zum Vergleich der operativen und konservativen Therapie der 3- und 4-Teile-Fraktur konnten nach Zuggurtungsosteosynthese keine besseren Ergebnisse als nach konservativer Therapie festgestellt werden. Auch Fjalestad et al. [3] kamen zu diesem Ergebnis. Die retrospektiven Untersuchungen von Ichmann et al. [11], Rasmussen et al. [23] sowie Zyto [34] zeigten befriedigende Ergebnisse bei konservativer Therapie dislozierter Frakturen des proximalen Humerus.

Durch die Einführung der winkelstabilen Plattenosteosynthese und der Nagelsysteme mit anatomiegerechter und stabiler Fixation der Verriegelungsschrauben im Humeruskopf hat eine neue Epoche der Frakturversorgung am proximalen Humerus begonnen. Mehrere Arbeitsgruppen konnten überzeugende Ergebnisse gerade auch bei der Versorgung der 3- und 4-Teile-Frakturen zeigen [7, 8, 13, 14]. Die Humeruskopfprothese ist beim Kalottenspaltbruch und auch bei der 4Teile-Fraktur eine Alternative und für verschiedene Autoren die Therapie der Wahl $[4,25,26,27]$. Nicht selten kann erst intraoperativ die Entscheidung getroffen werden, ob die Fraktur mit einem winkelstabilen Implantat stabil versorgt werden kann oder ob ein primärer Humeruskopfersatz notwendig ist.

Eine besondere Situation besteht beim sehr alten Menschen, ab einem Lebensalter ab 80 Jahren $[2,17,18,19]$. Aufgrund der demografischen Entwicklung wird dieser Bevölkerungsanteil in den nächsten Jahren stark zunehmen. Durch die regelmäßig anzutreffende, hochgradige Osteoporose ist auch die winkelstabile Plattenosteosynthese durch eine beachtliche Zahl an Komplikationen wie Schraubenperforation am Humeruskopf und Plattenausriss 


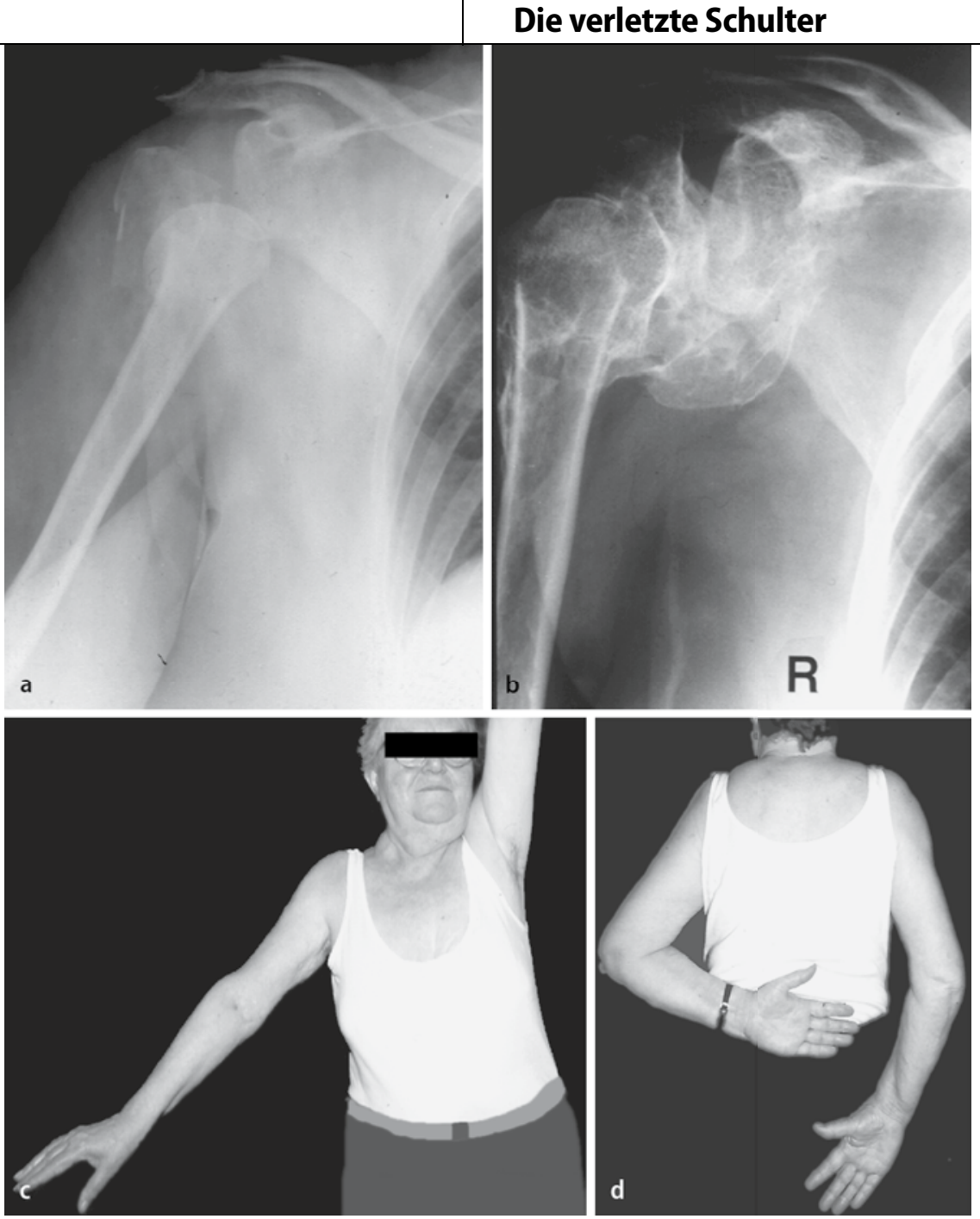

Abb. $5 \Delta$ Luxationsfraktur, konservative Therapie, a Röntgen zum Unfallzeitpunkt, b Röntgen nach 10 Jahren, c,d Schulterfunktion nach 10 Jahren

belastet [31]. Ein weiteres Problem sind häufige und schwere Begleitkrankheiten, die ein hohes Operationsrisiko bedingen, besonders auch für evtl. notwendige Revisionsoperationen $[10,31]$.

In der vorliegenden Studie zur konservativ-funktionellen Therapie nach Poelchen waren 3/4 der Patienten mit dem Behandlungsergebnis zufrieden oder sehr zufrieden, $2 / 3$ der Patienten hatten keine Schmerzen, und $3 / 4$ waren in ihrem täglichen Leben durch die Folgen der Fraktur nicht beeinträchtigt. Es zeigte sich, dass ältere Patienten häufig mit dem Behandlungsergebnis zufrieden waren und über keine relevante Behinderung durch die Folgen der proximalen Humerusfraktur klagten, obwohl objektiv eine deutliche Einschränkung der Schulterfunktion festzustellen war. Tingart et al. [30] konnten ebenfalls zeigen, dass besonders bei alten
Patienten eine Diskrepanz zwischen dem durch Constant- und Neer-Score gemessenen Behandlungsergebnis und der Patientenzufriedenheit besteht.

\section{Schlussfolgerung}

Wenn die Indikation zur konservativfunktionellen Therapie gestellt wird, sollte grundsätzlich durch eine dynamische Bildwandleruntersuchung die Stabilität der Fraktur beurteilt werden. Bewegt sich der Humeruskopf synchron zur passiven Bewegung des Arms mit, kann nach einer kurzen Ruhigstellung im GilchristVerband von etwa 1 Woche mit Pendelübungen begonnen werden. Zeigt sich eine instabile Fraktur und soll diese aufgrund von Kontraindikationen zur Operation konservativ behandelt werden, ist eine Ruhigstellung im Gilchrist-Verband von etwa 3 Wochen notwendig ( $\bullet$ Abb. 4) [16]. Erst dann kann mit Pendelübungen begonnen werden. Die Röntgenkontrolle a.-p. und transskapulär sollte nach 1 Woche im Stehen mit angelegtem GilchristVerband wiederholt werden, da die Fraktur in dieser Position verheilen wird [5]. Eine regelrechte konservative Therapie erfordert eine regelmäßige klinische und röntgenologische Kontrolle durch einen Unfallchirurgen, der mit dem regulären Heilungsverlauf einer proximalen Humerusfraktur vertraut ist.

Offensichtlich ist im angloamerikanischen Raum und in Skandinavien die konservative Therapie der proximalen Humerusfraktur weiter verbreitet. $\mathrm{Ob}$ die aktuellen Verhältnisse im deutschen Gesundheitssystem eine regelrechte Durchführung dieser aufwändigen, ambulanten Therapie überhaupt zulassen, muss bezweifelt werden. Wir sehen deshalb gerade beim alten Menschen mit einem biologischen Alter von über 80 Jahren eine Indikation zur konservativ-funktionellen Therapie der proximalen Humerusfraktur nach Poelchen neben der 1-Teil-Fraktur nach Neer nur bei hohem Operationsrisiko und bei Vorliegen einer 2- und 3Teile-Fraktur. Bei der 4-Teile-Fraktur, der Luxationsfraktur sowie beim Kopfspaltbruch ist bei konservativer Therapie nicht mit einem befriedigenden Ergebnis zu rechnen, weshalb diese nur beim Vorliegen von Kontraindikationen zur Operation konservativ behandelt werden sollten (• Abb. 5).

\section{Korrespondenzadresse}

\section{M.H. Amlang}

Klinik für Unfall-

und Wiederherstellungschirurgie,

Universitätsklinikum „Carl Gustav Carus",

Technische Universität Dresden,

Fetscherstraße 74, 01309 Dresden

Michael.Amlang@uniklinikum-dresden.de

Interessenkonflikt. Der korrespondierende Autor gibt an, dass kein Interessenkonflikt besteht.

\section{Literatur}

1. Clifford PC (1980) Fractures of the neck of the humerus: a review of the late results. Injury 12: 91-95

2. Einsiedel T, Becker C, Stengel D et al. (2006) Frakturen der oberen Extremität beim geriatrischen Patienten. Z Gerontol Geriatr 39: 451-461 
3. Fjalestad T, Stromsoe K, Blücher J et al. (2005) Fractures in the proximal humerus: functional outcome and evaluation of 70 patients treated in hospital. Arch Orthop Trauma Surg 125: 310-316

4. Gierer P, Simon C, Gradl G et al. (2006) Die Humeruskopfmehrfragmentfraktur - Versorgung mit einer Humeruskopfprothese? Orthopade 35: 834840

5. Habermeyer $P$ (1997) Die Humeruskopffraktur. Unfallchirurg 100: 820-837

6. Habermeyer P, Schweiberer L (1991) Oberarmkopffrakturen. Unfallchirurg 94: 438-446

7. Hessler C, Schmucker U, Matthes G et al. (2006) Ergebnisse nach Versorgung instabiler proximaler Humerusfrakturen mittels winkelstabiler Platte. Unfallchirurg 109: 867-874

8. Hessmann MH, Rommens PM (2001) Osteosynthesetechniken bei proximalen Humerusfrakturen. Chirurg 72: 1235-1245

9. Hoffmann R, Khodadadyan C, Raschke M et al. (1998) Die retrograde Markdrahtung bei proximaler Humerusfraktur des alten Menschen. Zentralbl Chir 123: 1232-1238

10. lannotti JP, Ramsey ML, Williams GR Jr et al. (2004) Nonprosthetic management of proximal humeral fractures. Instr Course Lect 53: 403-416

11. Ilchmann T, Ochsner PE, Wingstrand H et al. (1998) Non-operative treatment versus tenson-band osteosynthesis in three- and four-part proximal humeral fractures. Int Orthop 22: 316-320

12. Keene JS, Huizenga RE, Engber WD et al. (1983) Proximal humeral fractures. Orthopedics 6: 173178

13. Kettler $M$, Biberthaler $P$, Braunstein V et al. (2006) Die winkelstabile Osteosynthese am proximalen Humerus mit der PHILOS-Platte. Unfallchirurg 109: 1032-1040

14. Köstler W, Strohm PC, Südkamp NP (2003) Die proximale Humerusfraktur im hohen Lebensalter. Chirurg 74: 985-989

15. Koval KJ, Gallagher MA, Marsiciano JG et al. (1998) Functional outcome after minimal displaced fractures of the proximal part of the humerus. J Bone Joint Surg Am 79-A: 203-206

16. Kristiansen $B$, Angermann P, Larsen TK (1989) Functional results following fractures of the proximal humerus. Arch Orthop Trauma Surg 108: 339341

17. Leyshon RL (1984) Closed treatment of fractures of the proximal humerus. Acta Orthop Scand 55: 4851

18. Lill H, Josten C (2000) Proximale und distale Humerusfrakturen im hohen Alter. Orthopade 29: 327341

19. Lill $H$, Josten C (2001) Konservative oder operative Versorgung der Humeruskopffraktur beim alten Menschen? Chirurg 72: 1224-1234

20. Neer CS 2nd (1970) Displaced proximal humeral fractures: part I. Classification and evaluation. J Bone Joint Surg Am 52: 1077-1089

21. Plausinis D, Kwon YW, Zuckerman JD (2005) Complications of humeral head replacement for proximal humeral fractures. Instr Course Lect 54: 371380

22. Poelchen $R$ (1940) Selbstinnervationsbehandlung geschlossener Knochenbrüche und Verrenkungen, eine biologische Behandlungsart. Hippokrates, Stuttgart

23. Rasmussen S, Hvass I, Dalsgaard J et al. (1992) Displaced proximal humeral fractures: results of conservative treatment. Injury 23: 41-43

24. Resch H (2003) Humeruskopffrakturen. Unfallchirurg 106: 602-617
25. Robinson M, Page RS, Hill RMF et al. (2003) Primary hemiarthroplasty for treatment of proximal humeral fractures. J Bone Joint Surg Am 85-A: 12151223

26. Rüter A (2001) Indikation und Technik der Schulterendoprothese. Chirurg 72: 1246-1252

27. Schittko A, Ruter A (2003) Die proximale Humerusfraktur im hohen Lebensalter. Chirurg 74: 990-993

28. Schmidt M (2000) Die proximale Humerusfraktur operative versus konservative Therapie. Med. Dissertation, Technische Universität Dresden

29. Tingart M, Bäthis H, Bouillon B et al. (2001) Die dislozierte proximale Humersufraktur: Gibt es gesicherte Therapiekonzepte? Chirurg 72: 1284-1291

30. Tingart M, Bäthis $H$, Lefering R et al. (2001) Constant-Score und Neer-Score. Unfallchirurg 104: 1048-1054

31. Voigt C, Woltmann A, Partenheimer A et al. (2007) Komplikationsmanagement nach winkelstabiler Plattenosteosynthese am proximalen Humerus. Chirurg 78: 40-46

32. Weise K, Meeder P, Wentzensen A (1980) Indikation und Operationstechnik bei der Osteosynthese von Oberarmkopfluxationsfrakturen des Erwachsenen. Langenbecks Arch Surg 351: 91-98

33. Zuckermann JD, Koval KJ, Powell SE et al. (1995) One-part proximal humerus fractures: a prospective study of functional outcome. In: Vastamäki M, Galovaara P (eds) Surgery of the shoulder. Elsevier Science, Amsterdam New York

34. Zyto K (1998) Non-operative treatment of comminuted fractures of the proximal humerus in elderly patients. Injury 29: 349-352

35. Zyto K, Ahrengart L, Sperber A et al. (1997) Treatment of displaced proximal humeral fractures in elderly patients. J Bone Joint Surg Br 79-B: 411417 